\title{
The Reseach on Model Predictive Control for Linear Synchronous Motor of Maglev Train
}

\section{Li Yelei, Xu Hongze, Gegerile}

School of Electronic and Information Engineering, Beijing Jiaotong University, Beijing, China

\section{Email address:}

15120256@bjtu.edu.com (Li Yelei), hzxu@bjtu.edu.com (Xu Hongze), 15120243@bjtu.edu.com (Gegerile)

\section{To cite this article:}

Li Yelei, Xu Hongze, Gegerile. The Reseach on Model Predictive Control for Linear Synchronous Motor of Maglev Train. Science Discovery. Vol. 5, No. 2, 2017, pp. 77-85. doi: 10.11648/j.sd.20170502.11

Received: March 24, 2017; Accepted: April 18, 2017; Published: April 20, 2017

\begin{abstract}
The PID controller with an advantage of simple structure and easy extension is difficult to meet the requirements of the speed control system during the parameters variation and load disturbance. This paper presents a model predictive control(MPC) strategy for motor speed control based on analysis of the mathematical model of long stator linear synchronous motor(LSLSM), which utilizes move-blocking strategy to reduce the degrees of freedom of the controller by fixing the system input to keep it constant over several time. Then a low-computation MPC controller is designed for the sake of controller implementation in practice. Finally, the simulation experiments are conducted to verify the effectiveness of the proposed controller by using Matlab. Results show that the MPC controller effectively improved the dynamic performance and steady state accuracy of the system and the low-computation MPC reduced the computational complexity with a decline in accuracy.
\end{abstract}

Keywords: Long Stator Linear Synchronous Motor, Model Predictive Control, Move-blocking Strategy, Low-Computation

\section{磁悬浮列车直线同步电机的模型预测控制研究}

李烨雷, 徐洪泽, 格格日乐

电子信息工程学院, 北京交通大学, 北京, 中国

邮箱

15120256@bjtu. edu. cn（李烨雷），hzxu@bjtu. edu. cn（徐洪泽），15120243@bjtu. edu. cn（格格日乐）

摘要: PID速度控制器具有结构简单、易于实现等优点, 但是易受参数变化以及负载扰动影响, 因此难以满足高性能调 速系统的控制要求。本文在分析长定子直线同步电机数学模型的基础上，提出了一种针对电机速度控制的模型预测控 制方法, 同时应用输入分块化策略, 使若干时间段内的系统输入保持恒定值, 以此来降低控制器的自由度, 减少算法 计算时间，并且在此基础上设计了低计算量的模型预测控制器，从而提高了该算法硬件实现的可能性。最后，在Matlab 仿真环境中对所设计控制器进行仿真验证。仿真结果表明：相比PI控制器, MPC控制器有效地提高了系统的动态特性和 稳态精度。低计算量MPC有效地减少了控制器的计算量, 但是控制精度有所下降。

关键词: 长定子直线同步电机, 模型预测控制, 输入分块化策略, 低计算量 


\section{1. 引言}

目前为止，直线电机在工业、军事、民用以及其他领 域有着广泛的应用。它不需要任何的中间转换机构, 大大 提高了系统的传递效率, 又因为它在运动过程中传动装置 不受离心力的作用, 因此理论上来说速度不受限制, 具有 可靠性高, 机械损耗小, 噪声小, 结构简单以及散热能力 强等优点 $[1]$ 。

直线电机的一个重要应用场合便是磁悬浮列车。长定 子直线同步电机作为磁悬浮列车的直接动力来源, 研究其 牵引控制策略对于推动磁悬浮列车技术的发展具有重要 意义。然而, 交流电机是一个多变量、非线性、强耦合且 模型参数不确定以及具有端部效应等特点的系统, 其中包 含很多不确定因素, 很难对系统进行精准而有效地控制 [2]。针对直线同步电机的控制方法, 文献 [3]提出了一种 基于观测器的鲁棒控制方法。文献 [4]在电励磁同步电机 数学模型的基础上, 提出了一种基于PR调节器的电励磁同 步电机矢量控制方法。

模型预测控制 (Model Predictive Control, MPC) 是一种基于模型的计算机控制技术，它利用被控对象的动 态模型来预测系统未来的状态, 在每个控制周期内根据性 能指标函数决定最优的控制量, 使未来的一段控制周期内 被调量与经过柔化处理之后的期望值之间的误差为最小， 优点是其能够在控制律的设计中对未来变化的信息进行 最佳考虑 [5]。它采用多步预测、滚动优化和反馈校正等 控制策略，同时在每一个控制周期内不间断地将系统的实 际输出与预测模型输出之间的误差用来反馈校正, 所以可 以在一定程度上降低预测模型的误差和某些不确定性干 扰的影响, 因而控制效果好、鲁棒性强, 适用于控制不易 建立精确数学模型且比较复杂的控制对象 [6] [7], 恰好为 控制电机这样一个非线性、强耦合性的系统提供了一个方 案 [8]。文献 [9]将改进的增量型模型预测算法应用于电机 调速系统。文献 [10]考虑到模型预测控制算法计算量较大 的缺点, 提出了基于自动微分模型的改进预测控制算法。 文献 [11]提出了一种全速域自适应模型预测控制方法, 采 用离线初选和在线优化两个步骤完成权值系数配置, 保证 了MPC滚动优化的控制品质。文献 [12]提出了一种基于离 散扰动观测器的永磁同步电机复合预测控制策略, 内环电 流环由模型预测控制实现, 有效的提高了系统的动态响应 速度。文献 [13]提出一种改进的模型预测控制算法, 只需 一次预测即可选出最优电压矢量。针对传统模型预测控制 需要在每个采样周期对优化问题在线求解的特点, 本文引 入 “输入分块化策略” (move-blocking strategy), 通 过灵活的在线输入分块化来减少底层优化问题的自由度, 以此降低模型预测算法的复杂性和计算量 [14] [15]。

\section{LSLSM的数学模型建立及描述}

电机运行过程中由于磁路饱和及温度升高对电机参 数的影响, 同时励磁绕组和定子三相绕组之间, 三相绕组 相互间都存在电磁耦合, 因此想要建立其精确的数学模型 难度较大。为了简化过程, 作如下假设: a）假设定子三相绕组是对称的, 各绕组的匝数和电 阻均相等, 各相绕组轴线之间相差 120 度, 且参数 值完全一样;

b) 电机铁心导磁系数为无穷大, 且不计磁路饱和、 磁滞及浴流的影响, 假定磁路是线性的;

c）忽略励磁磁势的变化, 假定气隙恒定;

d）假定初级和次级表面无摩擦, 不计齿槽效应; 此时:

(1) 磁悬浮列车长定子直线同步电动机定子和转子绕 组的电压方程为:

$$
\left\{\begin{array}{l}
u_{d}=p \psi_{d}-\psi_{q} \omega_{r}+r_{s} i_{d} \\
u_{q}=p \psi_{q}+\psi_{d} \omega_{r}+r_{s} i_{q} \\
u_{f}=p \psi_{f}+r_{f} i_{f}
\end{array}\right.
$$

式中: $u_{d}$ - $\mathrm{d}$ 轴定子电压; $u_{q}-\mathrm{q}$ 轴定子电压; $u_{f}-\mathrm{d}$ 轴转子励磁电压; $i_{d}-\mathrm{d}$ 轴定子电流; $i_{q}-\mathrm{q}$ 轴定子电流; $i_{f}-\mathrm{d}$ 轴转子励磁电流; $\psi_{d}$ - $\mathrm{d}$ 轴定子磁链; $\psi_{q}-\mathrm{q}$ 轴定子 磁链; $\psi_{f}$ - $\mathrm{d}$ 轴转子励磁磁链; $r_{s}$ 一定子电枢绕组电阻; $r_{f}$ 转子励磁绕组电阻; $\omega$ 一电机转子电角速度; $p$ 一微分算 子;

(2) 磁链方程:

$$
\left\{\begin{array}{l}
\psi_{d}=L_{d} i_{d}+m_{a f} i_{f} \\
\psi_{q}=L_{q} i_{q} \\
\psi_{f}=m_{a f} i_{d}+L_{f} i_{f}
\end{array}\right.
$$

式中: $m_{a f}$ 一为励磁绕组和定子绕组轴线重合时的互 感系数; $L_{d}$ - 轴电枢绕组电感; $L_{q}$ 一 $\mathrm{q}$ 轴电枢绕组电感; $L_{f}$ 一转子励磁绕组 $d$ 轴电感;

(3) 电流方程:

$$
\left\{\begin{array}{l}
p i_{d}=\frac{u_{d}}{L_{d}}-\frac{r_{s}}{L_{d}} i_{d}+\omega_{r} \frac{L_{q}}{L_{d}} i_{q} \\
p i_{q}=\frac{u_{q}}{L_{q}}-\frac{r_{s}}{L_{q}} i_{q}-\omega_{r} \frac{L_{d}}{L_{q}} i_{d}-\omega_{r} \frac{m_{a f}}{L_{q}} i_{f}
\end{array}\right.
$$

长定子直线同步电机的转子运动速度和频率之间的 关系式为:

$$
\omega=\pi v / \tau_{s}
$$

式中: $v$ 一直线同步电机运行速度; $\tau_{s}$ 一直线同步电 机定子极距;

(4) 列车牵引力方程:

$$
\mathrm{F}_{x}=\frac{3}{2} P_{n} \frac{\pi}{\tau_{s}}\left(\psi_{d} i_{q}-\psi_{q} i_{d}\right)=\frac{3}{2} P_{n} \frac{\pi}{\tau_{s}}\left[\left(L_{d}-L_{q}\right) i_{d} i_{q}+m_{a f} i_{f} i_{q}\right]
$$

(5) 机械运动方程:

$$
p \omega=\frac{\pi}{\tau_{s}} \cdot \frac{F_{x}-F_{d}}{m}
$$

式中: $F_{d}$ 一负载阻力; $m$ 一列车质量; 
电机是一个非线性、强耦合系统, 为了简化分析过程, 根据 $i_{d}=0$ 的控制策略, 根据LSLSM在 $\mathrm{dq}$ 坐标系下的电流方 程, 建立直线同步电机的离散数学模型:

$$
p \omega=\frac{\pi}{\tau_{s}} \cdot \frac{F_{x}-F_{d}}{m}=\frac{\pi}{\tau_{s}} \cdot \frac{\frac{3}{2} P{ }_{n} \frac{\pi}{\tau_{s}} m_{a f} i_{f} i_{q}-F_{d}}{m}
$$

根据电流方程可知, 在状态方程 $i_{d}$ 中, $\omega i_{q}$ 的作用是 不可以忽略的。在方程 $i_{q}$ 中, 由于系统采用了 $i_{d}=0$ 的控 制策略, 因此 $\omega i_{d}=0$, 此时无耦合。在实际的控制系统 中, 输出信号 $u_{d}$ 和 $u_{q}, i_{d}$ 和 $\omega$ 均可以实际测量到。因此为 了将电机模型线性化, 将 $\omega i_{q}$ 作为一个测量干扰包含在模 型中。

基于上述的考虑, 将系统的状态变量设计为:

$$
x=\left[\begin{array}{llll}
i_{d} & i_{q} & \omega i_{q} & \omega
\end{array}\right]^{T}
$$

输入信号电压矢量

$$
u=\left[\begin{array}{ll}
u_{d} & u_{q}
\end{array}\right]^{T}
$$

运行阻力

$$
z=\left[\begin{array}{llll}
0 & 0 & 0 & F_{d}
\end{array}\right]^{T}
$$

由此得到电机的模型如下:

$$
\begin{gathered}
\dot{x}=\mathrm{A} x+B u+\mathrm{D} z \\
y=C x \\
A=\left[\begin{array}{cccc}
-\frac{r_{s}}{L_{d}} & 0 & \frac{L_{q}}{L_{d}} & 0 \\
0 & -\frac{r_{s}}{L_{q}} & 0 & \frac{m_{a f}}{L_{q}} i_{f} \\
0 & \frac{3}{2} P_{n}\left(\frac{\pi}{\tau_{s}}\right)^{2} m_{a f} i_{f} & 0 & 0 \\
0 & \frac{0}{m} & 0
\end{array}\right] \\
B=\left[\begin{array}{cc}
\frac{1}{L_{d}} & 0 \\
0 & \frac{1}{L_{q}} \\
0 & 0 \\
0 & 0
\end{array}\right] \quad C=\left[\begin{array}{llll}
0 & 0 & 0 & 1
\end{array}\right]
\end{gathered}
$$

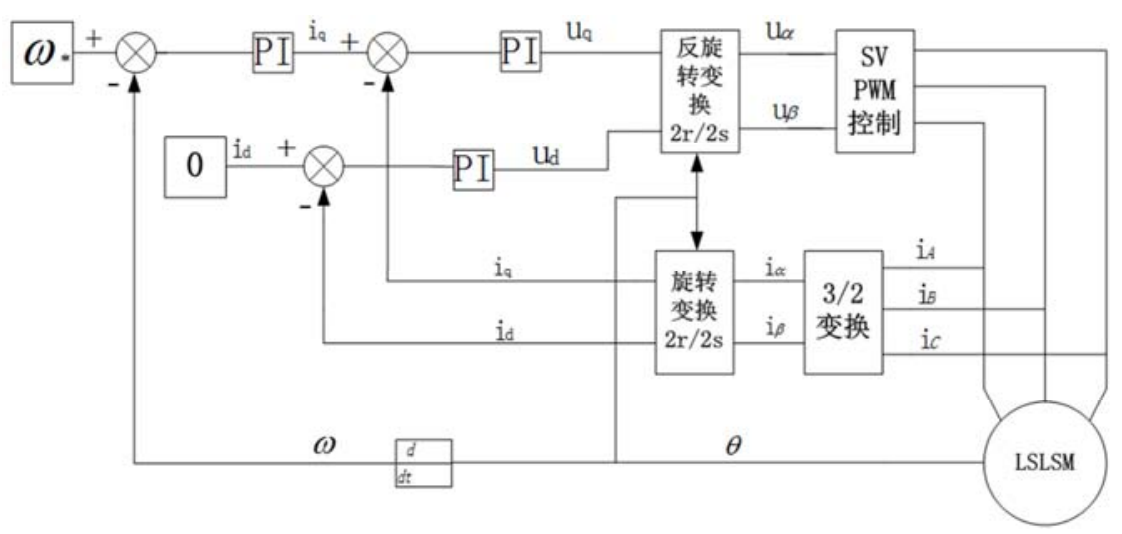

图1 长定子直线同步电机矢量控制系统结构图。

$$
\mathrm{D}=\left[\begin{array}{llll}
0 & 0 & 0 & \frac{\pi}{m \tau_{s}}
\end{array}\right]
$$

取采样时间为 $T_{S}$, 得到其离散化模型为:

$$
\begin{aligned}
& x(k+1)=A^{*} x(k)+B^{*} u(k)+D^{*} z \\
& y(k)=C^{*} x(k) \\
& A^{*}=\left[\begin{array}{ccccc}
1-T_{s} \frac{r_{s}}{L_{d}} & 0 & T_{s} \frac{L_{q}}{L_{d}} & 0 \\
0 & 1-T_{s} \frac{r_{s}}{L_{q}} & 0 & T_{s} \frac{m_{a f}}{L_{q}} i_{f} \\
0 & 0 & \frac{3}{2} P_{n}\left(\frac{\pi}{\tau_{s}}\right)^{2} m_{a f} i_{f} & 0 & 0 \\
0 & \frac{0}{m} & & 0
\end{array}\right] \\
& B^{*}=\left[\begin{array}{cc}
\frac{T_{s}}{L_{d}} & 0 \\
0 & \frac{T_{s}}{L_{q}} \\
0 & 0 \\
0 & 0
\end{array}\right] \quad C^{*}=\left[\begin{array}{llll}
0 & 0 & 0 & 1
\end{array}\right] \\
& D^{*}=\left[\begin{array}{llll}
0 & 0 & 0 & \frac{\pi}{m \tau_{s}}
\end{array}\right]
\end{aligned}
$$

\section{LSLSM矢量控制系统的MPC控制器设计}

在交流传动系统中, 由于转矩分量与励磁分量是耦合 在一起的, 如果想要获得高动态调速性能, 必须从电机的 动态模型出发, 目前广泛采用的方法是磁场定向控制理论, 直线同步电机亦然 [16]。

磁悬浮列车长定子直线同步电机本身具有一定的特 殊性: 其励磁磁极兼有悬浮磁极的作用, 但是励磁电流不 是由牵引控制系统来完成的, 因此从控制目标来看, 希望 定子电流的变化对列车悬浮力影响越小越好, 因此, 采用 恒定励磁且定子电流 $\mathrm{d}$ 轴分量 $i_{d}$ 恒为零的控制方法对实现 既定的控制目标最为合适。 
在Matlab/Simulink中, 由于没有现成的长定子直线同步电机的模块, 依据 $\mathrm{dq}$ 坐标系下的同步电机数学模型, 搭建 了长定子直线同步电机的Simulink仿真模块, 其结构图如下:

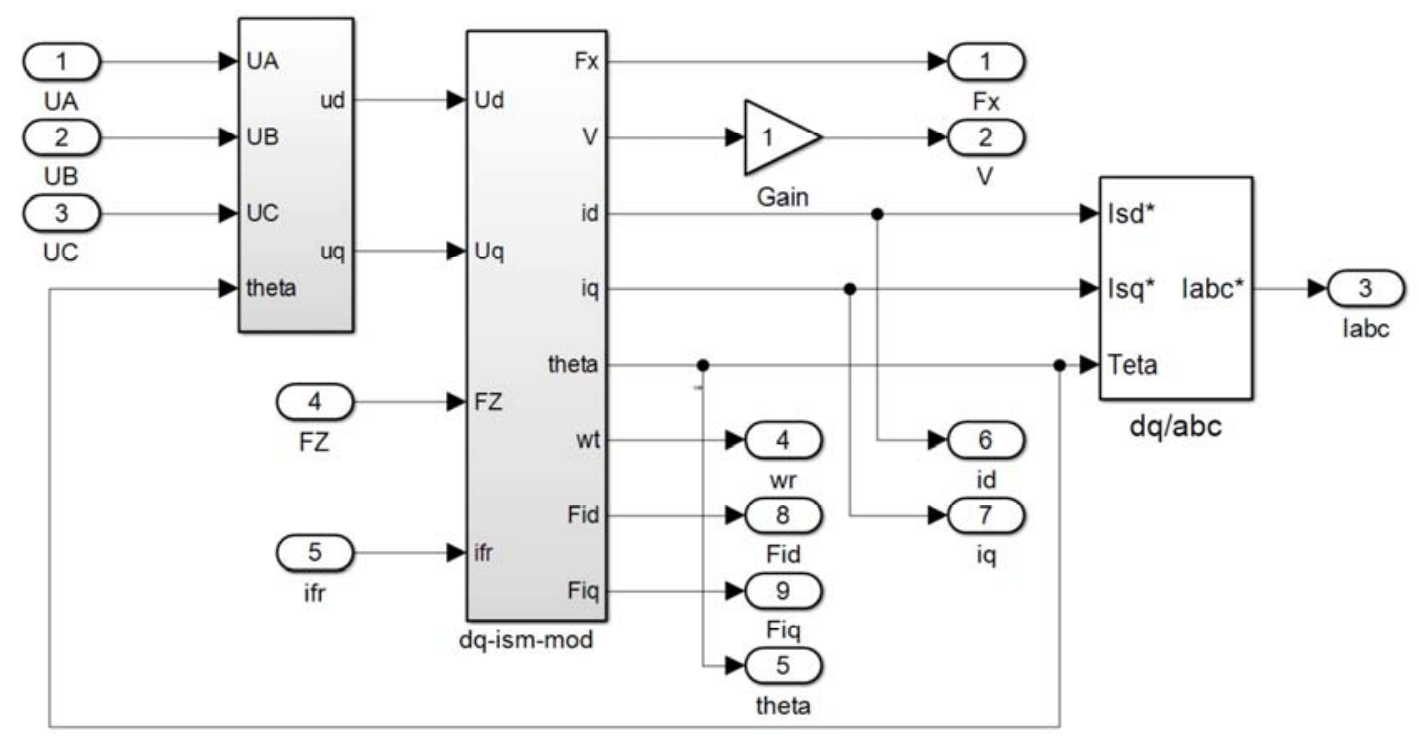

图2 长定子直线同步电机仿真模块。

其中, $\mathrm{dq}^{-1}-1 \mathrm{~s} 1 \mathrm{sm}-\mathrm{mod}$ 内部结构如下:

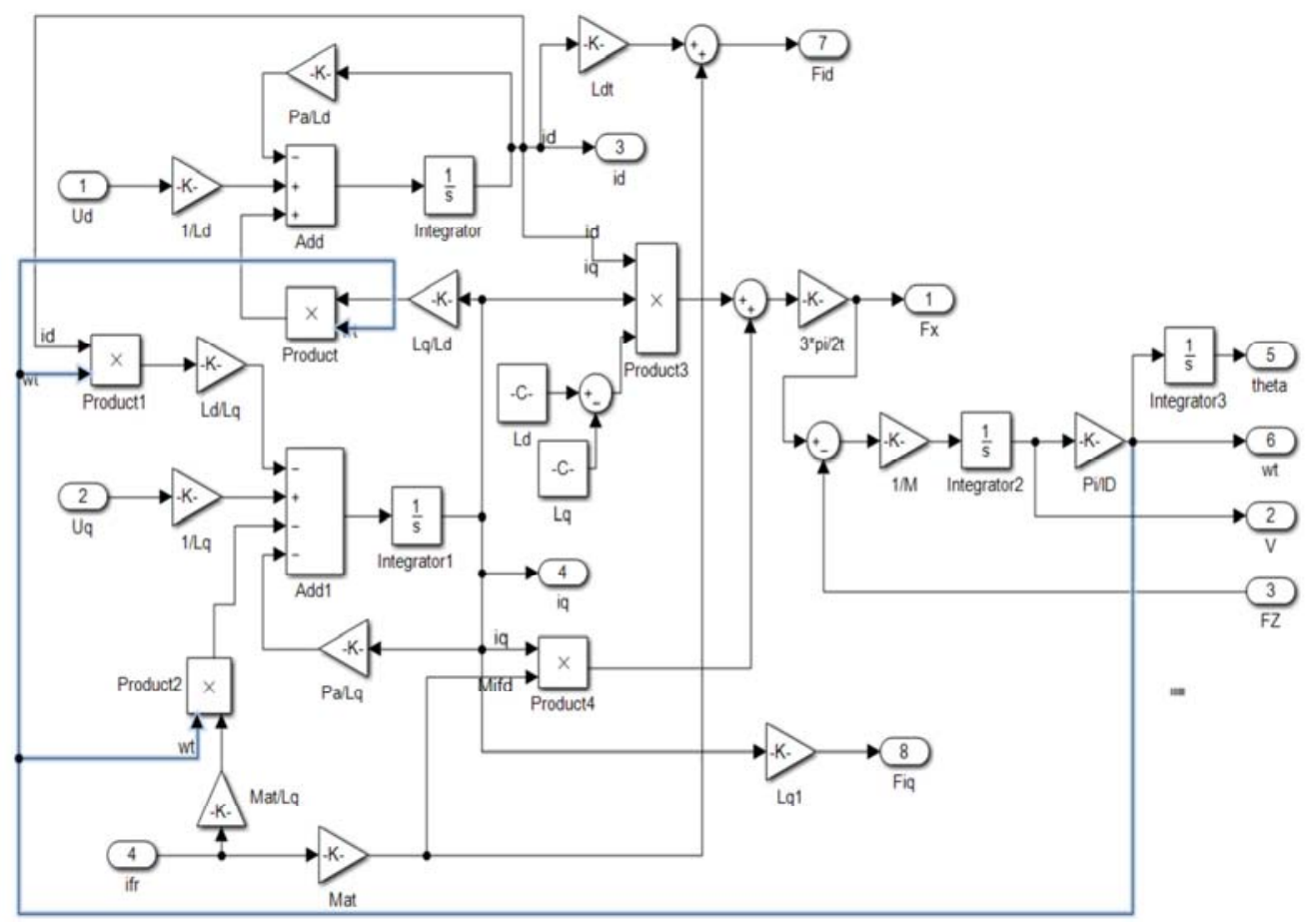

图3 长定子直线同步电机dq坐标系下仿真模块。

3. 1. 模型预测控制器的设计

\section{1. 1. 转速预测模型:}

由式 (7) 得:

$$
\frac{\omega(k+1)-\omega(k)}{T_{s}}=\frac{\pi}{\tau_{s} m}\left[\frac{3}{2} P_{n} \frac{\pi}{\tau_{s}} m_{a f} i_{f} i_{q}(k)-F_{d}\right]
$$

假设在采样时间 $T_{S}$ 内负载阻力不变, 那么由上式可得 上一个采样时刻的离散方程为:

$$
\frac{\omega(k)-\omega(k-1)}{T_{s}}=\frac{\pi}{\tau_{s} m}\left[\frac{3}{2} P_{n} \frac{\pi}{\tau_{s}} m_{a f} i_{f} i_{q}(k-1)-F_{d}\right]
$$

由式(15) (16) 得:

$$
\omega(k+1)=2 \omega(k)-\omega(k-1)+a \Delta i_{q}(k)
$$


式中：

$$
\begin{gathered}
\Delta i_{d}(k)=i_{q}(k)-i_{q}(k-1) \\
a=\frac{3}{2} P_{n} \frac{T_{s}}{m}\left(\frac{\pi}{\tau_{s}}\right)^{2} m_{a f} i_{f}
\end{gathered}
$$

于是, 在 $k$ 时刻给系统加入控制增量 $\Delta U(k)$, 如果该 增量在未来 $M$ 时刻都变化, 即 $\Delta u(k), \Delta u(k+1), \cdots$, $\Delta u(k+M-1)$, 则系统在未来 $P$ 个时刻的转速预测输出为 $(M \leq P)$ :

$$
\begin{gathered}
\omega_{m}(k+1 \mid k)=2 \omega_{p}(k \mid k)-\omega_{p}(k-1 \mid k)+a \Delta i_{q}(k) \\
\omega_{m}(k+2 \mid k)=2 \omega_{p}(k+1 \mid k)-\omega_{p}(k \mid k)+a \Delta i_{q}(k+1) \\
\vdots \\
\vdots \\
\omega_{m}(k+P \mid k)=2 \omega_{p}(k+P-1 \mid k)-\omega_{p}(k+P-2 \mid k)+a \Delta i_{q}(k+M-1)
\end{gathered}
$$

式中: $\omega_{p}(k \mid k)=\omega(k)$ 为 $k T$ 时刻的实际转速; $\omega_{p}(k-1 \mid k)=\omega(k-1)$, 为 $(k-1) T$ 时刻的实际转速;

\section{1.2. 反馈校正}

电机运行过程中受参数变化 (如电阻、电感以及磁链 的变化), 外部负载扰动以及非线性等因素的影响, 基于 预测模型计算得到的电机速度和实际的电机速度之间肯 定存在误差, 预测误差 $e(k)$ 为:

$$
e(k)=\omega(k)-\omega_{m}(k)
$$

其中:

$$
\omega_{m}(k)=2 \omega(k-1)-\omega(k-2)+a \Delta i_{q}(k-1)
$$

由于未来 $P$ 步模型预测中都会存在误差, 我们取未来 的预测误差为:

$$
e(k+P)=\cdots e(k+1)=e(k)
$$

用当前时刻实测误差对预测模型输出进行修正后，系 统在 $k+1$ 时刻的模型预测输出为:

$$
\omega_{p}(k+1)=\omega_{m}(k+1)+h e(k)
$$

即 $P$ 步模型预测误差为:

$$
E_{P \times 1}=\left[\begin{array}{llll}
h_{1} & h_{2} & \ldots & h_{P}
\end{array}\right]^{T} e(k)
$$

式中: $\omega_{p}(k+1)$ 一校正后的模型预测输出; $h$ 一由权 系数组成的 $P$ 维校正向量;

\section{1.3. 滚动优化}

在模型预测控制中, 控制的目的是使系统的期望输出 是从当前状态出发向设定值光滑过渡的一条参考轨迹, 我 们采用从现在时刻实际输出值出发的一阶指数形式来表 示参考轨迹为:

$$
\omega_{r}(k+i)=\left(1-\alpha^{i}\right) \omega_{s}+\alpha^{i} \omega(k) i=1,2, \ldots, P
$$

式中 $\omega_{s}$ 一输入设定值; $\alpha=\mathrm{e}^{-T / \tau}, \tau$ 为时间常数, $T$ 为 采样周期; $\omega_{r}(k+1)$ 一参考轨迹;

写成向量形式为:

$$
W_{r}=\left[\omega_{r}(k+1) \omega_{r}(k+2) \cdots \omega_{r}(k+P)\right]^{T}
$$

在控制过程中, 既希望未来时刻的预测输出尽可能地 接近参考轨迹, 又考虑到列车在牵引过程中不应该出现频 繁的加减速, 因此, 优化性能指标函数 $J$ 的选取可以采用 如下形式:

$$
\begin{gathered}
J=\sum_{i=1}^{P} q_{i}\left[\omega_{r}(k+i)-\omega_{p}(k+i)\right]^{2}+\sum_{j=1}^{M} r_{j}\left[\Delta i_{q}(k+j-\right. \\
1)]^{2} \quad r \geq 0
\end{gathered}
$$

将上式写成矩阵形式:

$$
J=\left(W_{r}-W_{m}-E\right)^{T} Q\left(W_{r}-W_{m}-E\right)+(\Delta U)^{T} R(\Delta U)
$$

其中:

$$
Q=\left[\begin{array}{cccc}
q_{1} & 0 & 0 & 0 \\
0 & q_{2} & 0 & 0 \\
0 & 0 & \ddots & 0 \\
0 & 0 & 0 & q_{P}
\end{array}\right]=\operatorname{diag}\left(\left[\begin{array}{llll}
q_{1} & q_{2} & \ldots & q_{P}
\end{array}\right]\right)
$$

矩阵 $W_{r}, W_{0}, W_{b}, E$ 在每个采样周期内都会实时更 新, 即所谓的滚动优化, 为了使性能指标函数取得极小值, 可通过极值必要条件 $\frac{d J}{d \Delta u_{M}(k)}=0$, 由此便可以得到控制增量:

$$
\Delta u_{M}(k)=\left(\mathrm{A}^{T} \mathrm{QA}+\mathrm{R}\right)^{-1} \mathrm{~A}^{T} \mathrm{Q}\left[W_{r}-W_{m}-E\right]
$$

上式给出了控制量 $\Delta u_{M}(k), \cdots, \Delta u_{M}(k+M-1)$ 的最 优值。但是系统并不把它们都当作应实现的解, 而只是取 其中的即时控制增量 $\Delta u_{M}(k)$, 构成实际的控制量。即在 $k T$ 时刻, 实际加在电机的控制量为:

$$
i_{q}(k)=\Delta i_{q}(k)+i_{q}(k-1)
$$

在下一个采样周期内, 采用相同的的优化过程求出 $\Delta u_{M}(k+1)$, 之后循环往复进行。 


\section{2. 输入分块化策略（move-blocking strategy）}

为了降低控制器计算复杂度, “move-blocking strategy”（输入分块化策略）广泛地应用于MPC控制器 的设计中 [17], 其基本原理是: 将输入系统的控制变量分 成若干组, 每组包含 $x$ 个控制变量, 每组内的控制变量在 $x$ 个采样周期内保持恒定, 从而避免了传统MPC控制器在每 个采样周期内均需要对滚动优化问题进行在线求解, 这无 疑会降低处理器的计算负荷 [18]。相比较于传统模型预测 控制, 基于 “输入分块化” 策略的模型预测控制 (MB-MPC) 速度控制器, 通过降低控制自由度来减小在线优化问题的 规模, 能在减少系统运算量的同时增强闭环系统的稳定性 [19]。

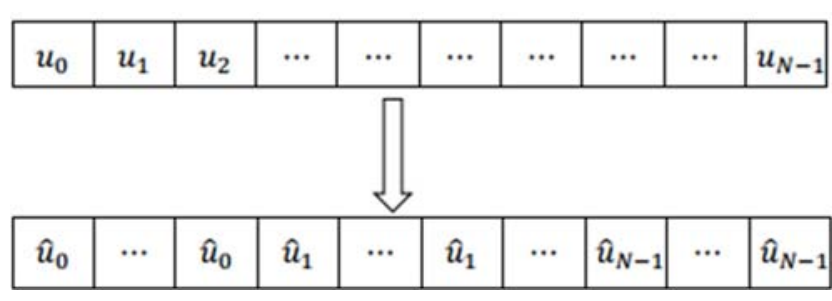

图4 输入分块化策略示意图。
将 “输入分块化” 策略用数学形式可表达为 [20]:

$$
U=\left(T \otimes I_{m}\right) \widehat{U}
$$

式中: 假设系统原来的控制量输入为

$U=\left[\begin{array}{llll}u_{0}^{T} & u_{1}^{T} & \cdots & u_{N-1}^{T}\end{array}\right]^{T}$; 输入分块化后的系统输 入变为 $\widehat{U}=\left[\begin{array}{llll}\hat{u}_{0}^{T} & \hat{u}_{1}^{T} & \cdots & \hat{u}_{M-1}^{T}\end{array}\right]^{T} ; \otimes$ 为克罗内克积运算 符; $T \in R_{N \times M}$ 为移动分块化矩阵。

\section{4. 仿真结果及分析}

仿真用的长定子直线同步电机参数如下 [21]:

表 1 长定子直线同步电机参数。

\begin{tabular}{llll}
\hline 名称 & 数值 & 名称 & 数值 \\
\hline 电压 & $400 \mathrm{~V}$ & 列车质量 & $1000 \mathrm{Kg}$ \\
极对数 & 8 & 定子直轴电感 & $0.1 \mathrm{H}$ \\
励磁电流 & $20 \mathrm{~A}$ & 定子交轴电感 & $0.02 \mathrm{H}$ \\
极距 & $0.3 \mathrm{~m}$ & 直轴互感 & $0.05 \mathrm{H}$ \\
定子电阻 & $0.5 \Omega$ & & \\
\hline
\end{tabular}

根据前面的描述, 我们在Matlab/simulink中搭建了 系统仿真模型, 其中转速外环的PI控制器可以被MPC控制 器替换, 整体结构如下:

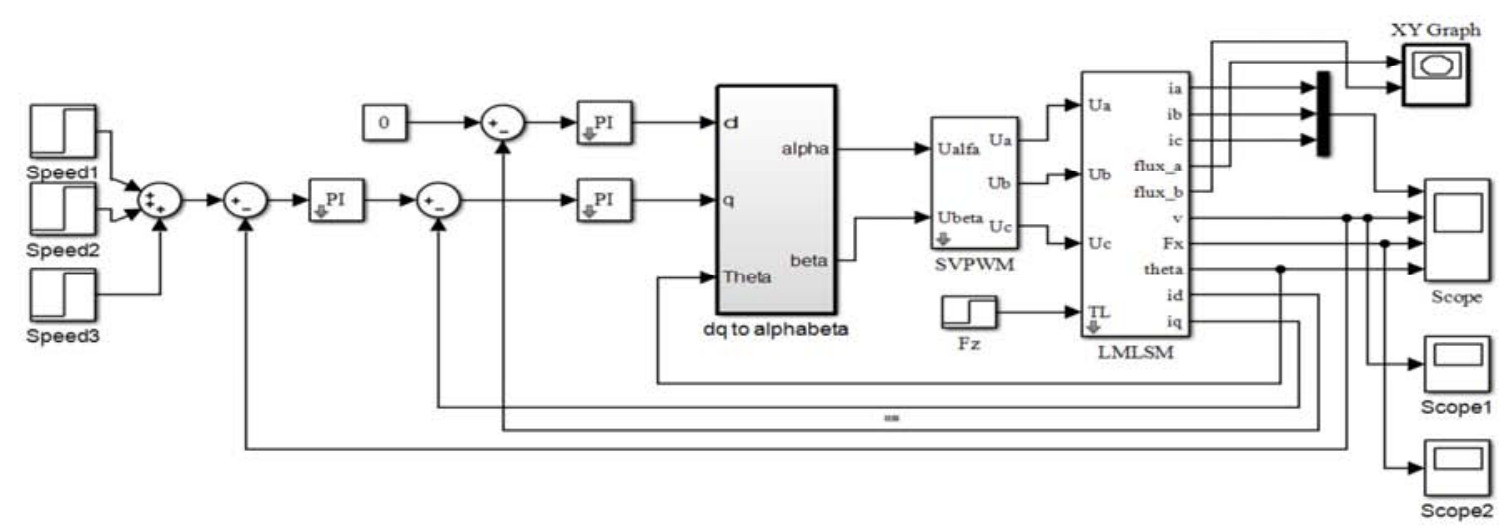

图5 直线同步电机调速系统仿真模型。

预测步长与控制步长的选取原则: 预测步长应该足够 大, 以保证跟踪效果和控制器的稳定性, 但是取值过大又 会使得算法的计算复杂度大大增加，这样不利于硬件实现。 控制步长则相对较小, 一般来说, $M<P$, 即控制量在 $k+M$ 时刻后不变。具体到磁悬浮列车牵引来说, 当列车 在加速减速阶段, 预测步长与控制步长应大一些, 这样有 利于列车的实时控制, 当列车在巡航阶段速度基本不变时, 预测步长与控制步长应小一些, 降低处理器负担。
仿真时, 设定电机空载启动, 给定速度为 $10 \mathrm{~m} / \mathrm{s}, 1$ 秒后速度变为 $20 \mathrm{~m} / \mathrm{s}, 2$ 秒后速度变为 $30 \mathrm{~m} / \mathrm{s}, 3$ 秒时突加负 载 $F_{d}=5000 \mathrm{~N}$, 观察不同情况下的仿真结果。

Case1: MPC算法中, 采样时间 $T_{S}=10^{-4} S$ 、预测步长 $P=8$, 控制步长 $M=6$, 权重矩阵均为相应维数的单位 矩阵, 误差校正系数 $h$ 均为 1 。

PI控制器和MPC控制器的仿真结果对比: 


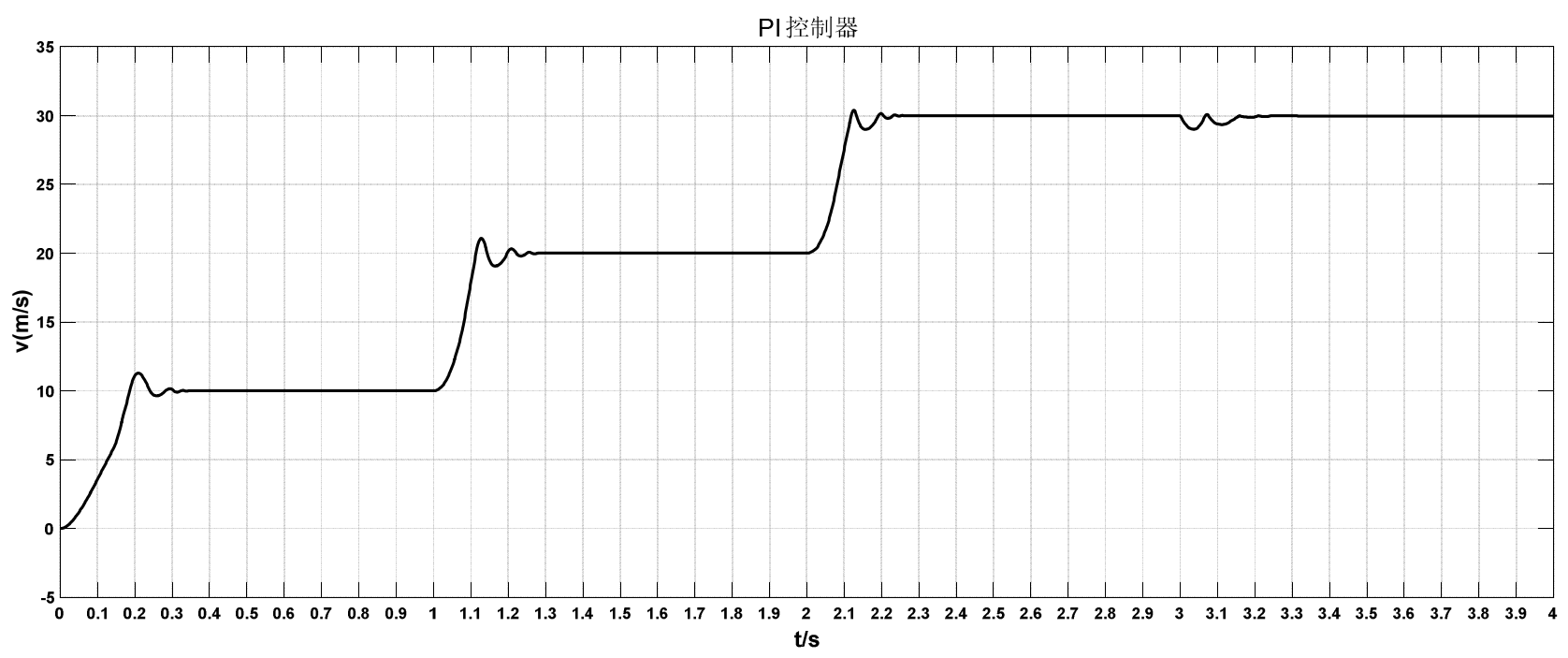

图6 PI控制器-速度曲线。

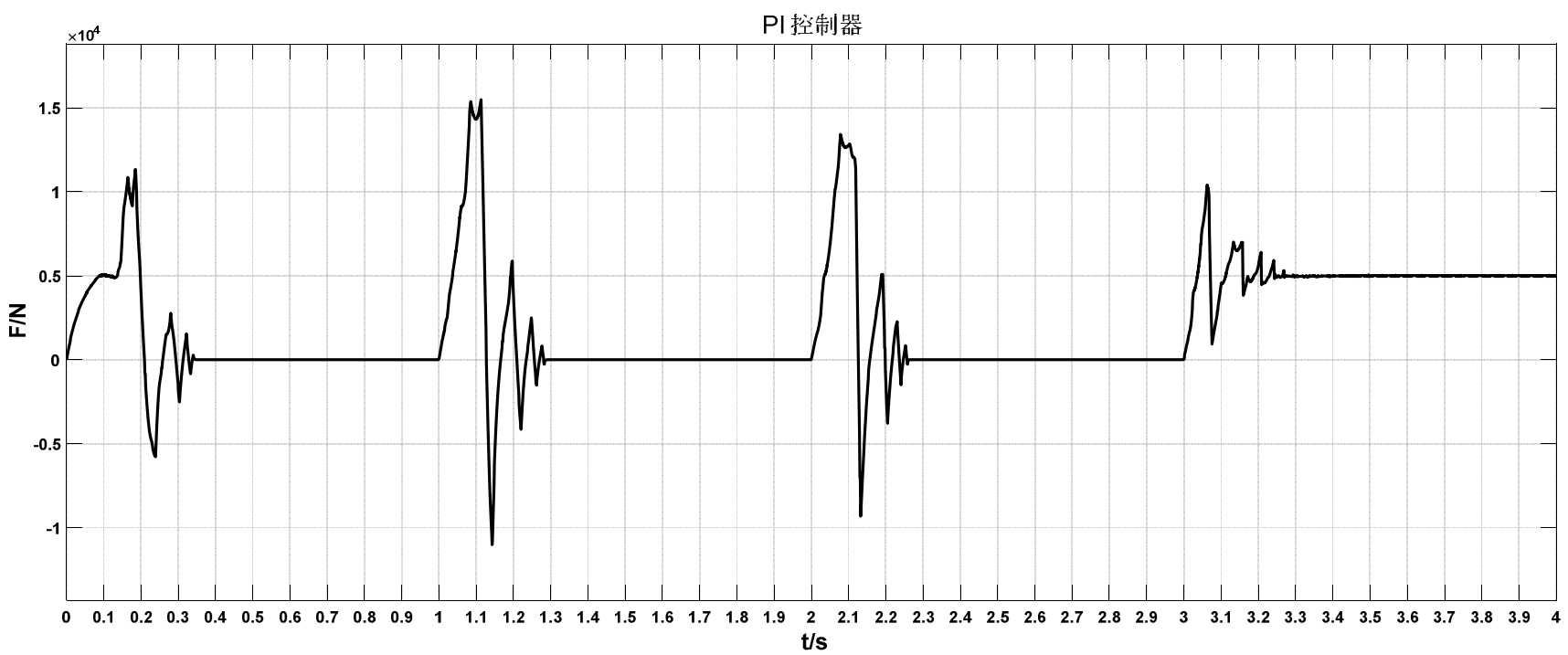

图7 PI控制器-牵引力曲线。

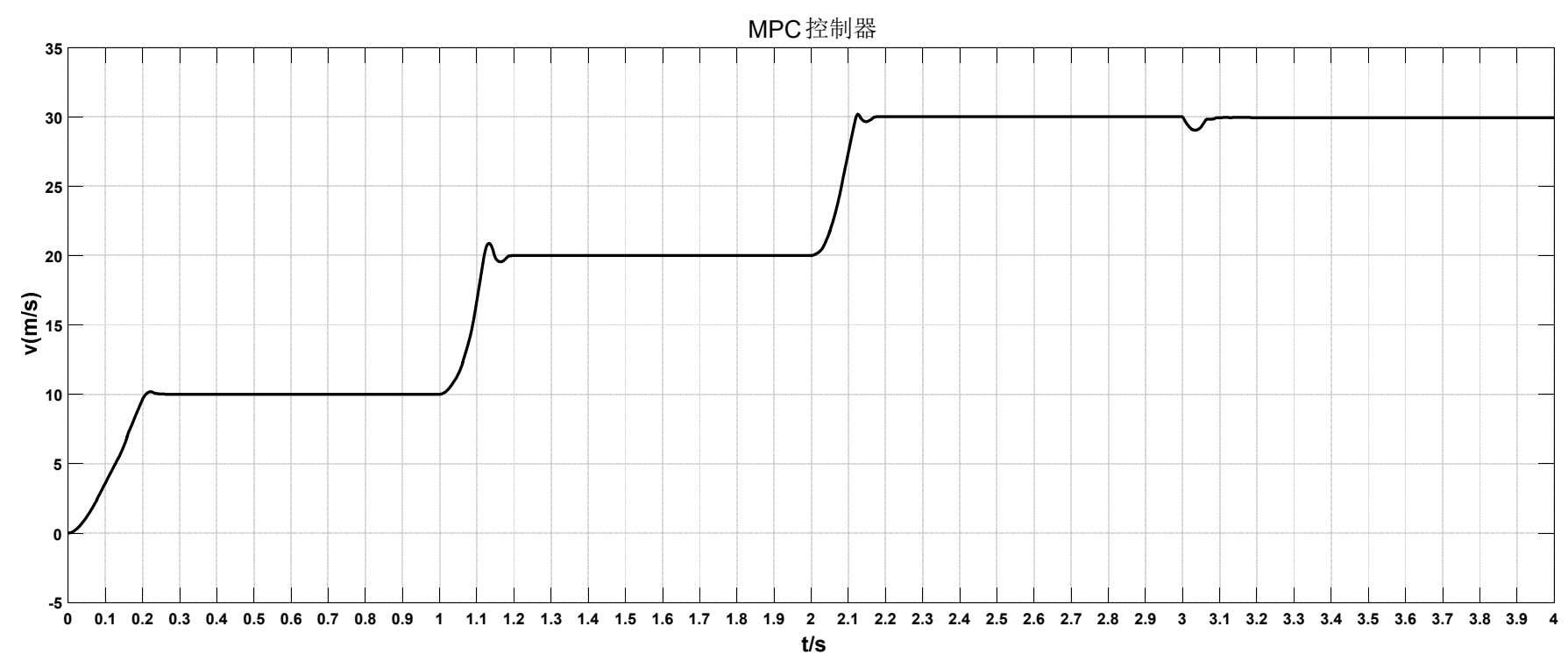

图8 MPC控制器-速度曲线。 


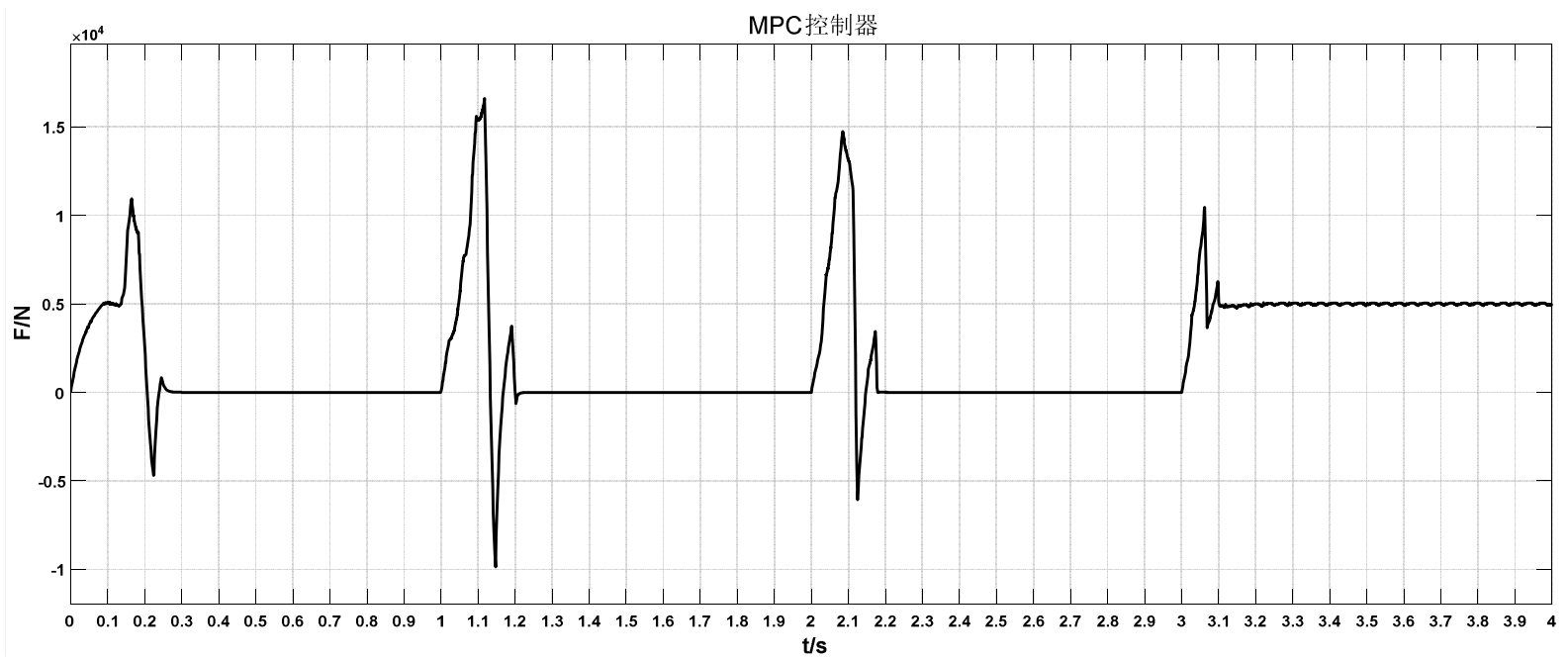

图9 MPC控制器-牵引力曲线。

仿真结果表明: 相对于PI控制器, MPC控制器能够较好的追踪给定速度, 且几乎不存在超调量, 牵引力的波动也较 小，在突加负载之后可以快速的达到新的稳定状态，动态性能良好。

Case 2: 应用输入分块化策略将输入控制量按照 $2: 2$ : 2的原则分块, MB-MPC控制器的仿真结果如下:

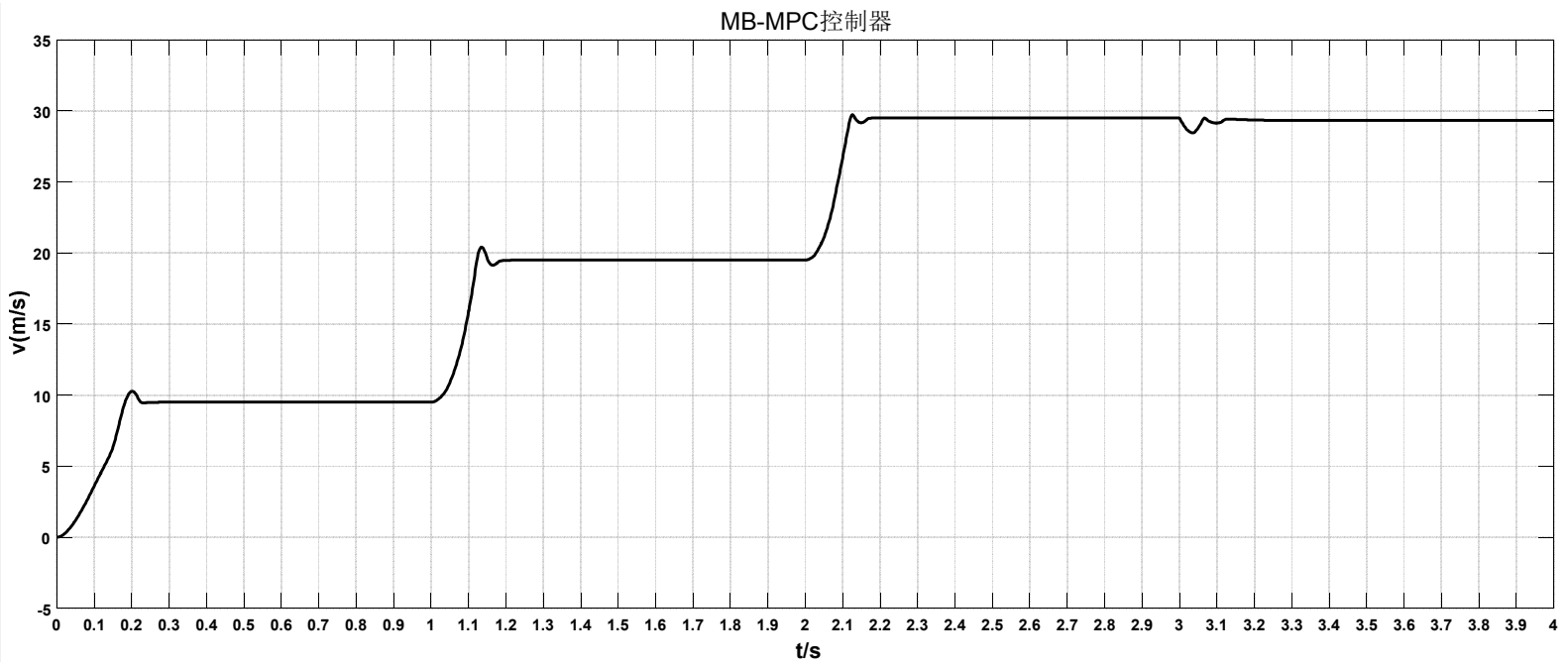

图10 MB-MPC控制器-速度曲线。

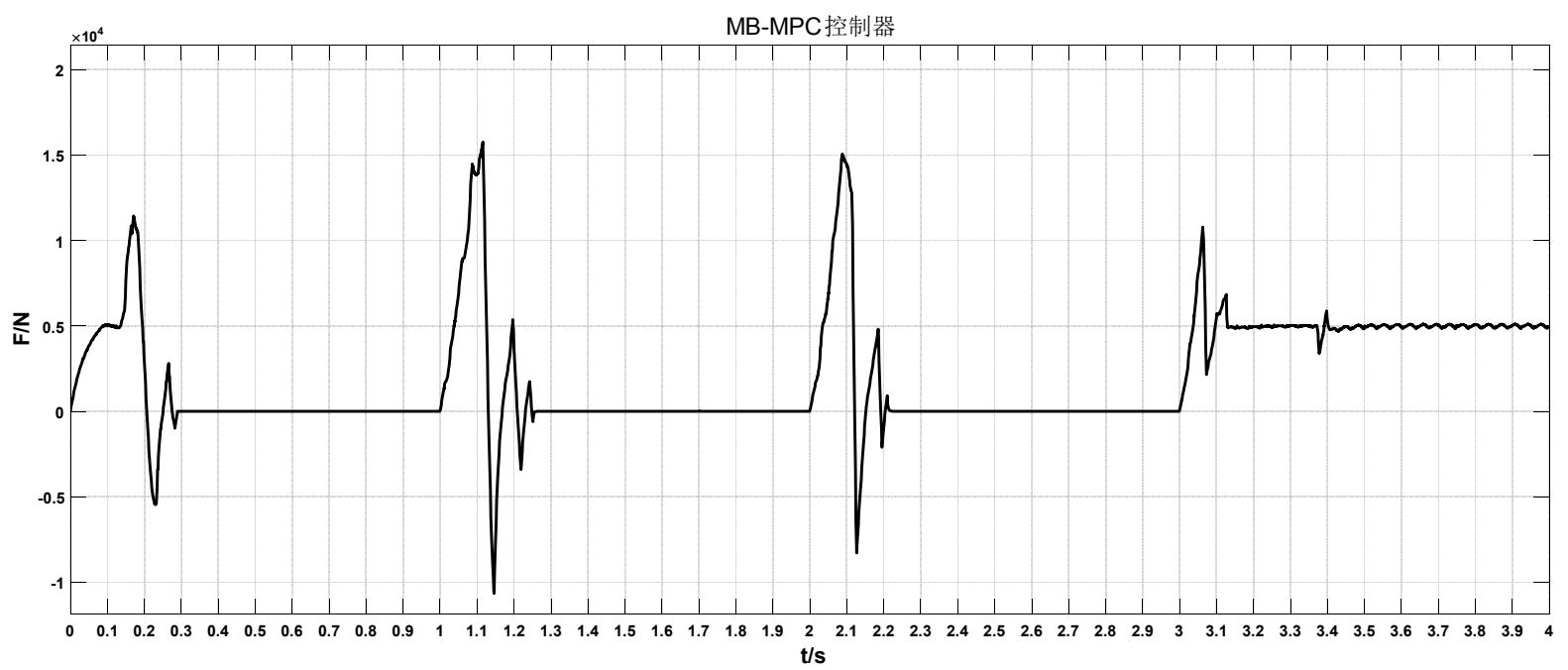

图11 MB-MPC控制器-牵引力曲线。 
仿真结果表明: MB-MPC控制器可以明显缩短系统的仿 真时间, 并且系统可以较快的达到给定速度, 但是系统却 存在明显的稳态误差, 且突加负载之后牵引力存在小幅度 的波动。

\section{5. 结论}

本文在分析长定子直线同步电机的数学模型的基础 上, 设计了基于模型预测控制算法的速度控制器, 同时引 入输入分块化策略, 有效地降低了控制器在线滚动优化过 程的计算量, 减少了计算时间。最后的仿真结果验证了相 对于传统PI控制器, MPC控制器对扰动具有较好的抑制作 用, 提高了系统的鲁棒性, 并且系统动态特性和稳态精度 均有提高。同时基于 “输入分块化” 策略的低计算量MPC 控制器有效地降低了计算复杂度, 提高了算法的实用性。

\section{参考文献}

[1] 叶云岳. 直线电机原理与应用 $[M]$. 北京: 机械工业出版社, 2000: 2-4。

[2] 李崇坚. 交流同步电机调速系统 [M]. 北京: 科学出版社, 2006:304-305。

[3] 柯兴利, 阳敏, 吴称列等. 一种直线同步电机的控制方法 [J]. 信息技术, 2017(1):164-169。

[4] 王志川, 姜林林. 基于PR调节器的电励磁同步电机系统仿真 研究 $[J]$. 微特电机, 2016, 44 (8) : 93-98。

[5] Valencia-Palomo G, Pelegrinis M, Rossiter J A, et al. A move-blocking strategy to improve tracking in predictive control[C] American: American Control Conference. IEEE, 2010:6293-6298.

[6] 舒迪前. 预测控制系统及其应用 [M]. 北京: 机械工业出版社, 2005: 60-61。

[7] 席裕庚. 预测控制 [M]. 北京: 国防科技出版社, 1993: 18-21。

[8] 葛宝明, 蒋静坪. 永磁同步电动机传动系统的模型算法控制 [J]. 中国电机工程学报, 1999, 19 (10): 27-31。
[9] 刘兵, 吴根忠. 增量型模型算法控制在PMSM速度控制中的应 用 $[J]$. 机电工程, 2009, 26 (11) : 18-20。

[10] 高丽媛, 卢达, 赵光宙等. 应用自动微分的永磁同步电机预 测控制 [J]. 电机与控制学报, 2012, 16(10):38-43。

[11] 刘向昕, 曹晓冬, 谭国俊等. 电励磁同步电机全速域自适应 模型预测控制 [J]. 电工技术学报, 2017, 32 (4) : 112-122。

[12] 吴麟. 基于扰动观测器的永磁同步电机复合预测控制 [J]. 计算机仿真, 2017 (1) : 356-359。

[13] 张永昌, 杨海涛, 魏香龙. 基于快速矢量选择的永磁同步电 机模型预测控制 [J]. 电工技术学报, 2016, 31 (6) :66-73。

[14] W H Chen, D J Balance, P J Gawthrop. Optimal control of nonlinear systems: a predictive control approach [J]. Automatica, 2003, 39(4), 633-641.

[15] Yongbin Wang, Hui Lin. Design of Model Predictive Control for Permanent Magnet Synchronous Motors: International Conference on Intelligent Computing and Cognitive Informatics (ICICCI 2010) [C]. Kuala Lumpur: IEEE, 2010: 223-226.

[16] 阮毅. 电力拖动自动控制系统-运动控制系统 [M]. 北京: 机 械工业出版社, 2009: 155-156。

[17] NL Ricker. Use of quadratic programming for constrained internal model control [J]. Industrial \& Engineering Chemistry Process Design and Development, 1985, 24(4) :925-936.

[18] Shekhar R C, Manzie C. Optimal move blocking strategies for model predictive control [J]. Automatica, 2015, 61 (C) :27-34.

[19] Shekhar R C, Maciejowski J M. Robust variable horizon MPC with move blocking $[\mathrm{J}]$. Systems \& Control Letters, 2012, $61(4): 587-594$.

[20] Tim Schwickart. A Flexible Move Blocking Strategy to Speed up Mode1-Predictive Control while Retaining a High Tracking Performance: European Control Conference [C]. Denmark. IEEE, Denmark. IEEE, 2016:764-769.

[21] 罗云飞. 基于DSP的直线同步电机磁场定向控制系统 [D]. 成 都:西南交通大学, 2004:36-37。 